\title{
A Modified TV Tuner for Spectrum Sensing in TV UHF Bands
}

\author{
Sameh Tahseen \\ Higher Institute for Engineering \\ Thebes Academy \\ Elec. \& Comm. Eng. Dept. \\ Maadi, Cairo, Egypt
}

\author{
Mohamed Abouelatta \\ Faculty of Engineering \\ Ain Shams University \\ Elec. \& Comm. Eng. Dept. \\ Abbassia, Cairo, 11517, Egypt
}

\author{
Abdelhalim Zekry \\ Faculty of Engineering \\ Ain Shams University \\ Elec. \& Comm. Eng. Dept. \\ Abbassia, Cairo, 11517, Egypt
}

\begin{abstract}
A novel system containing low cost TV box has been designed to sense the UHF TV band spectrum. Microcontroller chip is programmed using micro Pascal software to control the TV tuner for scanning the TV band. The implemented system has been used to measure the utilization of the UHF TV band covering the frequency range from 470 to $860 \mathrm{MHz}$ in Cairo (EGYPT). The sensing output data has been recorded and analyzed using suitable software. The obtained results demonstrate significant amount of spectrum available for deployment of cognitive radio systems and IEEE 802.22 WRAN standard in the UHF TV broadcasting band
\end{abstract}

\section{General Terms}

A new methodology for detecting UHF TV broadcasting band, TV tuner, UHF TV Spectrum usage.

\section{Keywords}

Cognitive Radio, Spectrum sensing, TV tuner, UHF TV band spectrum usage.

\section{INTRODUCTION}

Wireless communication systems were grown significantly. However this growth is faced by limits, because wireless communications is using the radio spectrum which is a finite resource. Cognitive Radio (CR) concept appeared trying to saturate this famine.CR is an intelligent adaptable wireless communication system that is aware of its surrounding environment and uses the methodology of understanding-bybuilding to learn from the environment and adapt its internal parameters, in order to detect the presence of the primary user signal, and detects the unused spectrum and shares it without harmful interference to other users. It determines which portion of the spectrum is available and detects the presence or absence of licensed users when a user operates in licensed band. In all countries, the government regulates the usage of the frequency spectrum by national regulatory institutions like the Federal Communications Commission (FCC) in the USA. In Egypt, the National Telecommunication Regulatory Authority (NTRA) is the governmental authority that administer the telecommunication sector. FCC coordinated allocating frequency bands and issuing exclusive licenses to systems within a geographical area while regulating other systems with respect to these bands.

\subsection{SPACE SPECTRUM}

\subsubsection{TV Band Spectrum}

In November 2008 the United States FCC issued a R\&O on the unlicensed use of TV white space spectrum [1]. To operate in TV white space, there are requirements needed based on cognitive radio technology including location awareness and spectrum sensing. There are a number of other requirements intended to provide protection for the licensed services that operate in the TV bands. These requirements impose technical challenges for the design of devices operating in TV white space spectrum. FCC set rules managing the operation of the TV band devices (TVBDs).

There are two classes of TV band devices: fixed and personal/portable. The shorter term portable will be used for the personal/portable devices. These portable devices are divided into Mode I and Mode II devices.

Fixed devices are permitted to transmit up to $30 \mathrm{dBm}$ ( 1 watt) with up to $6 \mathrm{dBi}$ antenna gain, while portable devices are permitted to transmit up to $20 \mathrm{dBm}(100 \mathrm{mw})$ with no antenna gain. Fixed devices are permitted to use a higher gain antenna as long as the transmit power is decreased $\mathrm{dB}$-for- $\mathrm{dB}$ for any antenna gain above $6 \mathrm{dBi}$.

The Very High Frequency (VHF) channels (2-13) and the Ultra High Frequency (UHF) channels (14-51) are TV channels. However, there are restrictions on which channels are permissible for use by TVBDs. Channels (3-4) in the VHF channels and channels (36-38) in the UHF channels are not permitted for fixed devices. Fixed devices are forbidden to use channel (3-4) to prevent interference with external devices (e.g. DVD players) when they connect to a TV utility. Portable devices are not permitted at all in the VHF band. However they are permitted in UHF band except channels (14-20) and channel 37.Channel 37 is a protected channel, used for radio astronomy measurements. Finally, Portable devices are not permitted on channels (14-20) since, some of those channels are used for public safety applications [2].

\subsection{SPECTRUM SENSING}

Sensing the spectrum hole is an important requirement of cognitive radio network. In general, spectrum holes are categorized into two types, spatial spectrum holes and temporal spectrum holes. A spatial spectrum holes is unoccupied band by the primary user (PU) therefore the secondary users (SUs) can use it. A temporal spectrum hole is unoccupied band by the PU during the sensing time. Hence, the SUs can occupy this band in the current time slot $[3,4]$.

The spectrum holes are classified into three broadly defined types [5, 6]: Black spaces, which are occupied some of the time with high power RF signal interference. Gray spaces, which are partially occupied with low power signal. White spaces, which are free with RF interference except for white Gaussian noise. 


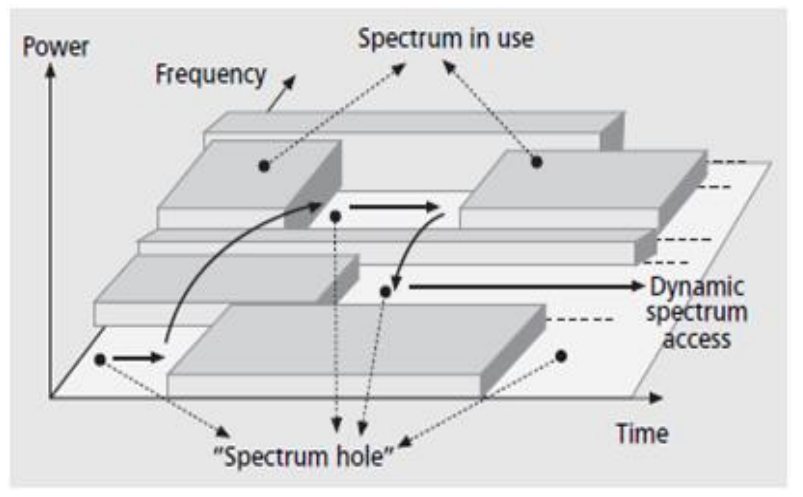

Fig 1: Spectrum Holes

The Gray and White spaces can be used by SU (unlicensed operators), but Black spaces cannot be used because of the interference that will be caused to the PUs. IF one or more of PUs back to reuse his band (White or Gray space), the CR moves to another available spectrum hole as shown in Figure 1 [4], and that's why Cognitive Radio considered as intelligent adaptable wireless communication system used to achieve the mission of spectrum utilization regarding causing interference between licensed users and unlicensed (portable) users, by using one or more of the spectrum sensing techniques which is discussed in next section.

The characteristics and operation of the primary users inherently dictates the secondary users' opportunity spectrum access. The primary users also have the privileged access to the frequency band. An acceptable level of interference must be guaranteed by the secondary users of their ability to share the spectrum with the primary users.

Recently Spectrum sensing has been developed, where it is defined and various aspects of spectrum sensing task are mentioned and discussed.

Tian Z. and G.B. Giannakis. (2006) [7] have developed a wavelet approach to efficient spectrum sensing of wideband channels. They have developed a couple of wideband spectrum sensing techniques based on the local maxima of the wavelet transform modulus and the multi-scale wavelet products, which provided an effective radio sensing architecture to identify and locate spectrum holes in the signal spectrum.

Zhi, Q. et al. (2008) [8] have introduced a novel wideband spectrum sensing technique, called multiband joint detection, which jointly detects the signal energy levels over multiple frequency bands rather than consider one band at a time.

Nekovee, M. (n.d.). (2010) [9] have reviewed the state-ofthe-art in technology, regulation and standardization of cognitive access to TVWS and examined the spectrum opportunity and commercial use cases associated with this form of secondary access.

Dzulkifli, M. et al. (2011) [10] have presented the spectrum occupancy of the UHF TV band in the frequency range from 470 to $798 \mathrm{MHz}$ in an outdoor environment of a suburban area in Johor Bahru, Malaysia.

Nishant Dev Khaira and Prateek Bhadauria (2012) [11] have investigated the optimization problem of detection efficiency under the constraint of detection probability.
Fawzy, A. et al. (2015) [12] have designed and implemented a wideband front end spectrum sensor built from commercial of the shelf components, they have provided the sensor front end architecture, component details, circuit design, and implementation.

\subsubsection{CLASSIFICATION OF SPECTRUM SENSING TECHNIQUES}

Spectrum sensing techniques are classified into three main types as shown in Figure 2, Non Cooperative sensing (Transmitter Detection), Cooperative sensing and Interference based sensing. Non Cooperative sensing is further classified into energy detection, matched filter detection and cyclostationary feature detection [3].

a) Non-cooperative detection

Is based on the detection of the weak signal from a primary transmitter. In primary transmitter based detection techniques, a cognitive user determines signal strength generated from the primary user. In this method, the location of the PUs are not known to the cognitive users because there is no signaling between the primary users and the cognitive users.

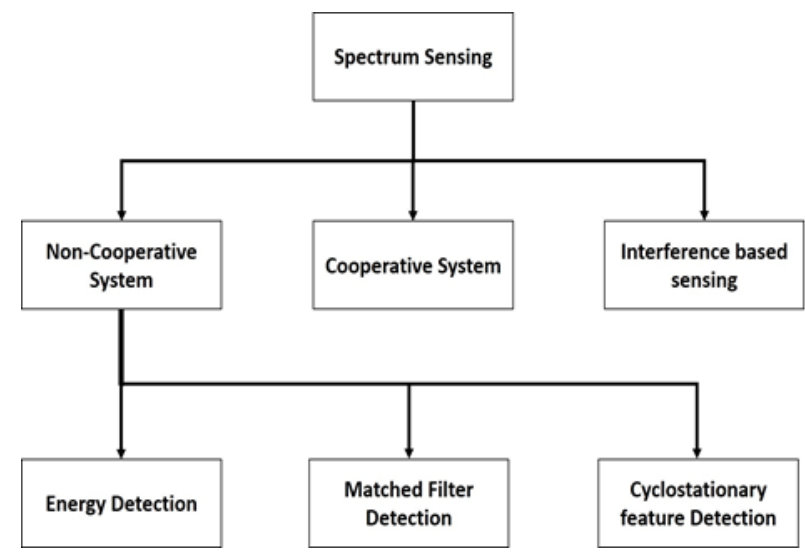

Fig 2: Classification of spectrum sensing techniques

Transmitter detection can be defined as follows:

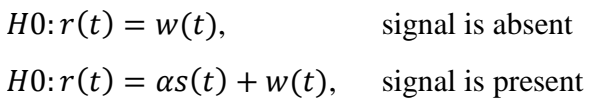

Where $r(t)$ is the signal received by the cognitive user, $s(t)$ is the transmitted signal of the primary user, $w(t)$ is the AWGN (Additive White Gaussian Noise) and $\alpha$ is the amplitude gain of the channel. $\mathrm{H} 0$ is a null hypothesis, $\mathrm{H} 1$ is an alternative hypothesis.

b) Cooperative or collaborative detection

In this technique cognitive radio users are in cooperation, where information from multiple users is incorporated for primary user detection.

In this work we aim to achieve TV UHF band sensor using novel setup containing the low cost commercial TV box set controlled by a programmed microcontroller chip IC. In which the TV spectrum band can be swept within a short time interval compared to other costly sensors. Adjustable frequency division can be obtained. 


\section{SYSTEM SETUP}

The reduced block diagram of the spectrum sensor is shown in Figure 3. It is composed of the antenna, the tuning and sensing unit as well as the spectrum analyzer. Figure 4 shows the detailed block diagram of system.

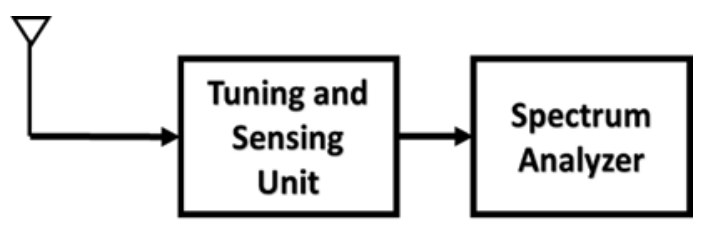

Fig 3: Reduced Spectrum sensor block diagram

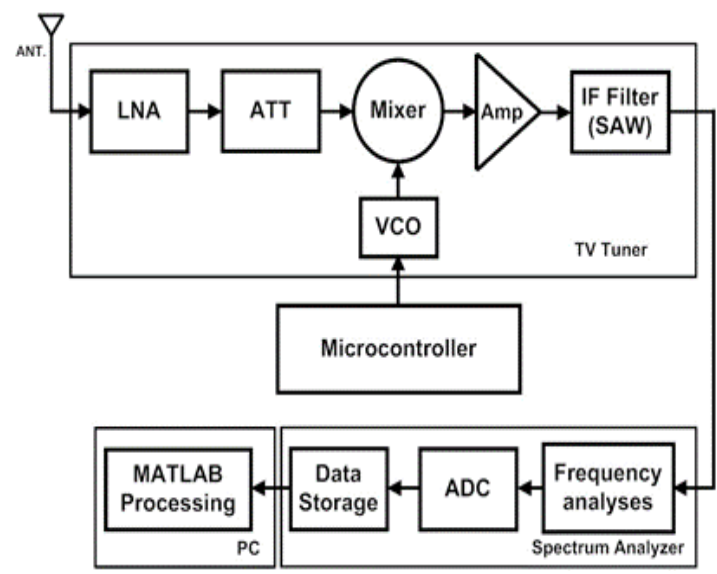

Fig 4: Detailed Block diagram of the system

The tuning and sensing unit is purposely built from a TV UHF band receiver that consists of omnidirectional antenna, a commercial TV set box, and a microcontroller chip.

The radio frequency signal is captured by the antenna and fed to the TV tuner. This signal is then amplified and converted down to an Intermediate Frequency IF using internal mixer whose local voltage controlled oscillator frequency is controlled by a microcontroller chip IC. The microcontroller is programmed such that the voltage controlled local oscillator frequency generates frequencies that is when mixed with the input RF signals frequencies produces the intermediate frequency IF signal of the TV. Then the IF signal is fed after amplification to the spectrum analyzer. By using RS-232 cable and software, the output of the spectrum analyzer is sent to laptop where it is stored processed using Matlab and displayed.

The spectrum data gathered over time is evaluated to estimate the UHF TV band utilization.

A photo of the built spectrum sensing set up according to the block diagram in Fig. 4, is shown in Fig. 5

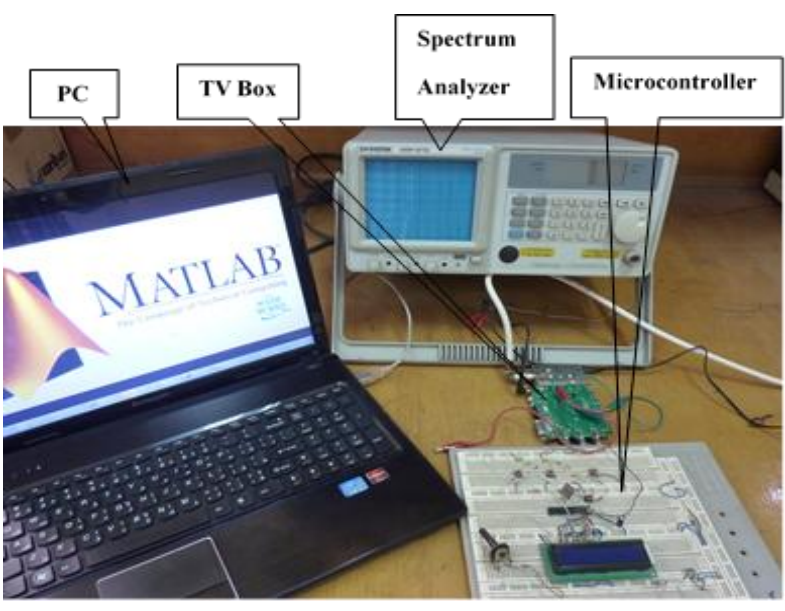

Fig 5: UHF TV band spectrum sensing setup

The used tuner module is modified to scan automatically the UHF TV band spectrum. It is so that older tuners modules are controlled by discrete analog voltages applied on the varactor diodes of the voltage controlled oscillator (VFO). This has been replaced with Inter-Integrated Circuit (I2C) controlled tuner. I2C will be discussed in the next section.

Figure 6 shows the tuner TNF 8327-DF3 pin out, which is built in the used TV box. Pin1 is Automatic Gain Control AGC voltage, Pin 2 not connected, Pin 3 and Pin 4 are I2C bus, where Pin 3 is Serial Clock (SCL) and Pin 4 is Serial Data (SDA), Pin 5 and Pin 6 are biasing voltages $5 \mathrm{~V}$ and 33V respectively, and finally Pin 7 is IF output.

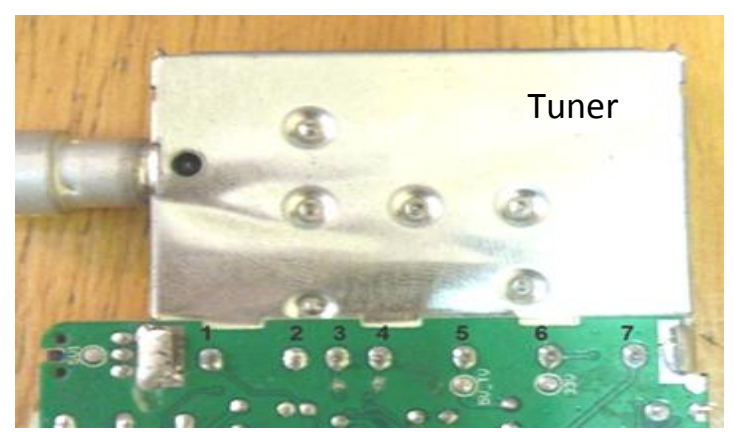

Fig 6: TV Tuner Pinout

\subsection{Microcontroller programing}

Phillips introduced the I2C bus for mass-produced items such as television and audio equipment, the I2C bus is a bidirectional two wire serial bus that provides a communication link between integrated circuits.

The I2C paths have been cut to disconnect them from the internal control of scanning and are connected to the new programmed microcontroller I2C pins to control the frequency scanning. This programmed microchip together with the voltage controlled local oscillator of the TV work as a frequency synthesizer.

\subsubsection{I2C write mode $(R / W=0)$}

Using the internal tuning IC (SN761678) datasheet we found the parameters and details that helped us to program the microcontroller chip. Table 1 shows the write data format. N14 to N0 are Programmable counter set bits. The oscillator frequency FLO is given by 


$$
F L O=F_{r} \times 8 \times N,
$$

where

$$
N=\mathrm{N} 14 \times 2^{14}+\mathrm{N} 13 \times 2^{13}+\cdots+\mathrm{N} 1 \times 2+\mathrm{N} 0,(2)
$$

and $F_{r}$ is the reference frequency

$$
F_{r}=4 \mathrm{MHz} / \text { (Reference Divider) }
$$

$\mathrm{CP}$ is Charge pump current set bit, $\mathrm{CP}=1$. T2, $\mathrm{T} 1$ and $\mathrm{T} 0$ are Test bits and are set to 0,0 and 1 respectively as a normal mode. RSA and RSB are Reference divider ratio selection bits as shown in Table 2. By selecting a reference divider as 640 , 1024 , or 512 , from equation (3) we get reference frequencies as $50,31.25$, or $62.5 \mathrm{KHz}$ respectively. BS4 to $\mathrm{BS} 1$ is band switch byte, selecting BS4 = 1 will choose UHF band.

The local oscillator frequency is mixed with RF input signal to generate the IF signal. This output is amplified and fed to the spectrum analyzer.

Figure 7 shows a photo of the implemented microcontroller and its complete circuit diagram is depicted in Figure 8.

The circuit diagram is drawn using NI Multisim circuit design software. Figure (8-a) shows the programmed microcontroller chip IC connected to an LCD display to project the output data as well as, the setting mode and the frequency. Fig 8-b shows the circuit diagrams of the supporting circuits of the microcontroller system.

The Three buttons for mode, frequency count-up and frequency count-down design are shown in Figure (8-b). Figure (8-c) shows the programming circuit using ICD connector connected to the burner. Switch Debouncer circuit is shown in Figure (8-d). Finally Figure (8-e) shows the source power fed to SCL and SDA paths.

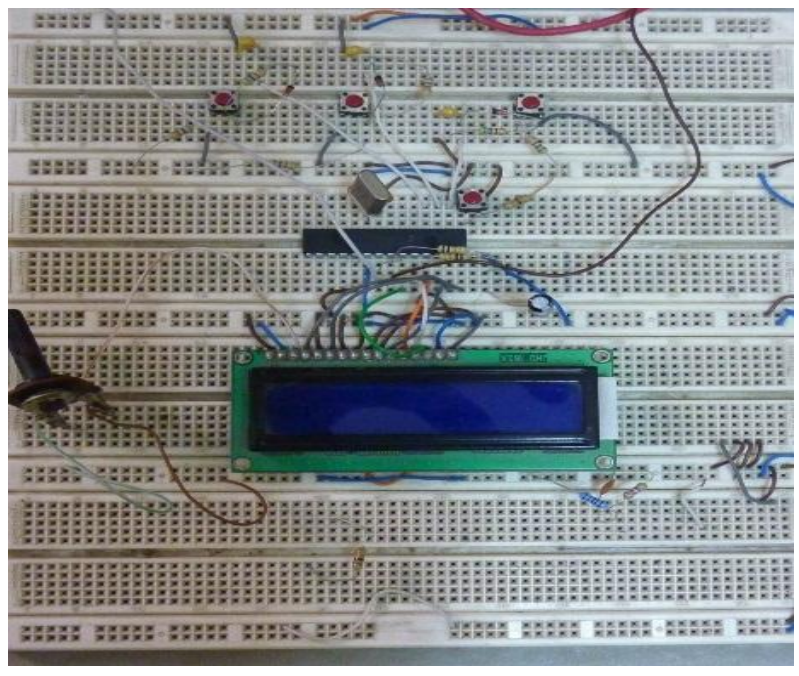

Fig 7: The controller unit circuit board

\subsection{SPECTRUM ANALYZER}

A high performance spectrum analyzer GW Instek is used, with frequency range of $(150 \mathrm{KHz}$ to $1 \mathrm{GHz})$, reference level range of $(-30 \mathrm{dBm}$ to $+20 \mathrm{dBm})$, bandwidth range is $3 \mathrm{k}, 30 \mathrm{k}$, $220 \mathrm{k}$ and $4 \mathrm{MHz}$ with accuracy $15 \%$. It supports standard RS232 interface for data exchange, which ease connecting to PC. An Input/output software is used to establish communication between the spectrum analyzer and the laptop which was used for automatic configuration of the instrument and data saving.

The received spectrum sensing analog data was digitized using the ADC with 10 bits resolution in the spectrum analyzer by spectrum analyzer software in the laptop included for displaying, saving and printing results via the standard RS232 interface .

The measurement has taken place at the ASUFE (Ain Shams University Faculty of Engineering) buildings which roughly $4.5 \mathrm{~km}$ away from the TV transmitter station building at Masbero in Cairo.

Table 1: write data format for the tuner

\begin{tabular}{|c|c|c|c|c|c|c|c|c|c|}
\hline & MSB & & & & & & & LSB & \\
\hline Address byte (ADB) & 1 & 1 & 0 & 0 & 0 & 0 & 1 & R/W=0 & A \\
\hline Divider byte 1 (DB1) & 0 & N14 & N13 & N12 & N11 & N10 & N9 & N8 & A \\
\hline Divider byte 2 (DB2) & N7 & N6 & N5 & N4 & N3 & N2 & N1 & N0 & A \\
\hline Control byte (CB) & 1 & CP & T2 & T1 & T0 & RSA & RSB & 0 & A \\
\hline Band switch byte (BB) & X & X & X & X & BS4 & BS3 & BS2 & BS1 & A \\
\hline
\end{tabular}

Table 2: TV Ratio Select Bits

\begin{tabular}{|c|c|c|}
\hline RSA & RSB & REFERENCE DIVIDER RATIO \\
\hline $\mathrm{X}$ & 0 & 640 \\
\hline 0 & 1 & 1024 \\
\hline 1 & 1 & 512 \\
\hline
\end{tabular}




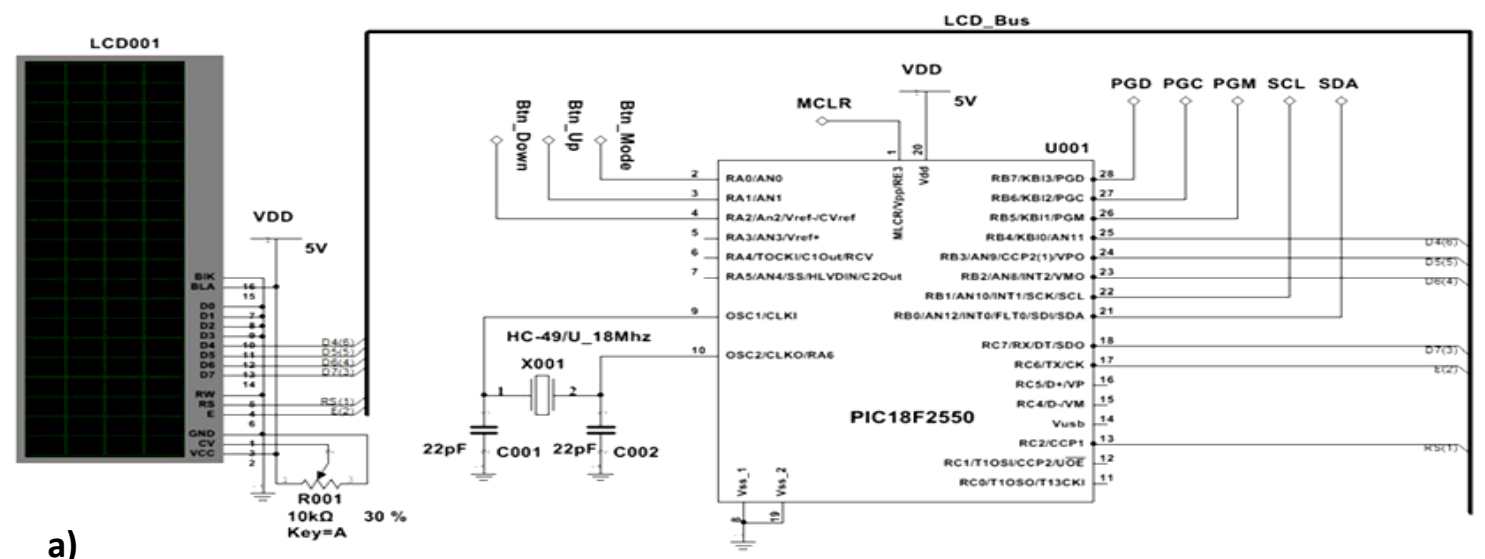

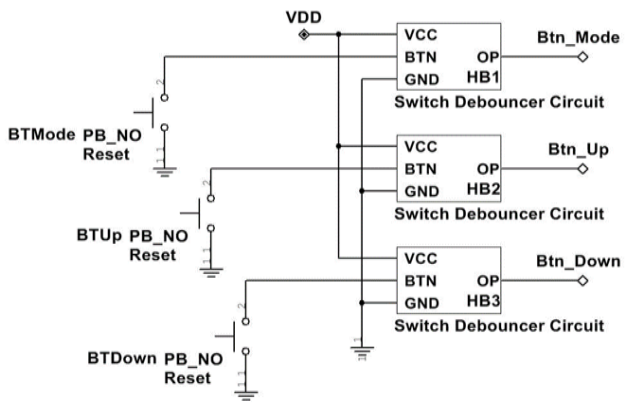

b)

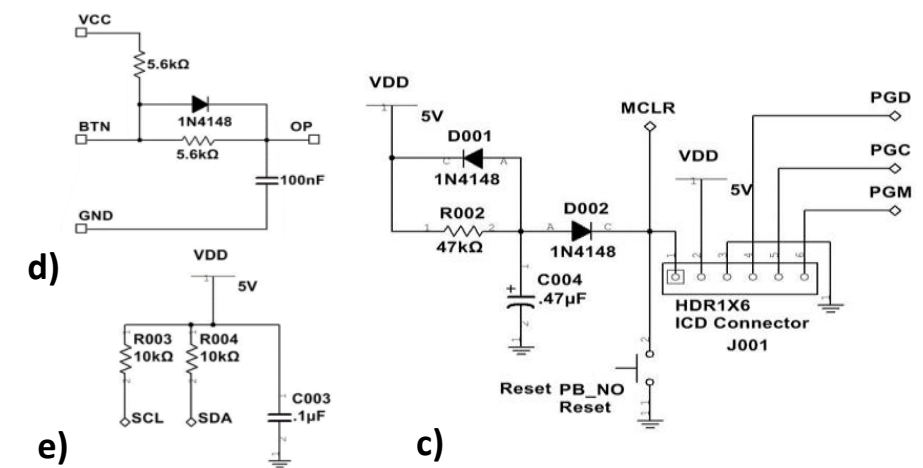

Fig 8: System circuit diagram

Sweeping of the whole TV band lasts around 2 minutes while choosing frequency reference of $62.5 \mathrm{kHz}$. This is the highest scanning speed to get reproducible spectrum analysis result. Setting the spectrum analyzer with RBW $220 \mathrm{kHz}$, span 5 MHz/Div. and $0 \mathrm{~dB}$ REF LVL. We observed the spectrum in the intended band for total time of 9 hours with 2 minutes interval for one sweep.

\section{SPECTRUM OCCUPANCY RESULTS AND ANALYSIS}

The measurements results are shown in Figure 9, and the calculated spectrum occupancy are shown in Figure 10 and Figure 11. The first graph shows the average received power versus frequency. The second and third graphs is a plot showing the spectrum occupancy versus frequency.

Figure 10 shows the spectrum occupancy of the UHF TV band with a threshold of $-67 \mathrm{dBm}$. As seen from the second graph, the spectrum utilization is $14.82 \%$, therefore the occupancy is high at the lower region of the band and becomes less towards higher frequencies.

Figure 11 shows the spectrum occupancy of the UHF TV band with a threshold of $-60 \mathrm{dBm}$. As shown in the second graph as well, the spectrum utilization is $1.57 \%$, therefore the occupancy is very high from 470 to $700 \mathrm{MHz}$, but it appears to have less utilization above the $700 \mathrm{MHz}$ region. It is also seen from the figures that there is an additional radio frequency activities beside the TV channels, mostly because of local wireless microphone. Comparing the two figures, threshold of $-67 \mathrm{dBm}$ seems to give a higher occupancy. Using the higher value of the threshold seems to indicate a possibility of false detection, i.e. the frequency is currently occupied but considered as vacant. It is however, both figures seems to possess an abundant of white spaces, especially in the $700 \mathrm{MHz}$ region, which could provide possible opportunity for the deployment of IEEE 802.22 WRAN. The heavy utilization in the UHF TV broadcasting is because of the close distance between the measurements site and the Egyptian TV transmitter (Masbero) in Cairo. There are nine television stations operating in Egypt. Transmission frequencies of these stations are shown in Table 3 and plotted in Figure 9.

Table 3: TV channels in Egypt

\begin{tabular}{|c|c|}
\hline Channel No. & $\begin{array}{c}\text { Center frequency } \\
\text { (MHz) }\end{array}$ \\
\hline 22 & 479 \\
\hline 26 & 511 \\
\hline 28 & 526 \\
\hline 30 & 543 \\
\hline 34 & 575 \\
\hline 38 & 607 \\
\hline 40 & 623 \\
\hline 46 & 671 \\
\hline
\end{tabular}

Current TV broadcasting uses analogue transmission. The transition to digital terrestrial television (DTTV) is currently underway and expected to be completed by the end of 2015, nationwide (worldwide completion of the digital transition is at the end of 2015 as well). DTTV systems deploy digital technology instead of the conventional analogue technology for the delivery of terrestrial free to air or subscription television services [10].

TV analogue system requires nine frequency channel (each with a Band Width (BW) of $8 \mathrm{MHz}$ ) in order to transmit all of the TV channels resulting in $72 \mathrm{MHz}(9 \times 8 \mathrm{MHz})$ of $\mathrm{BW}$. By 
switching to DTTV, these nine TV channels could be accommodated in much smaller bandwidth. Once the completion of the digital transition is finished, more white spaces are expected to be available for further exploitation [10].

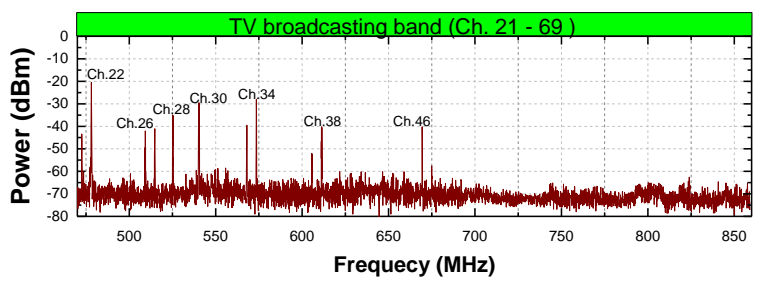

Fig 9: Measured frequency spectrum sensed in TV band

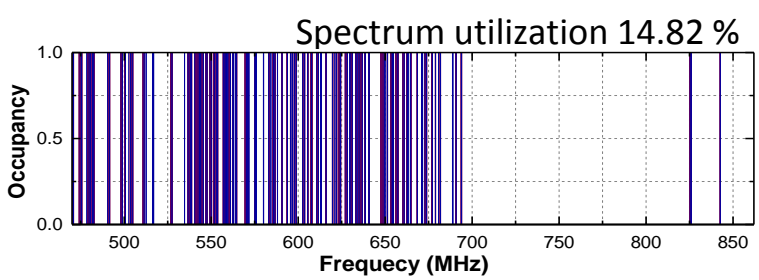

Fig 10: Calculated spectrum occupancy of UHF TV band with threshold $-67 \mathrm{dBm}$

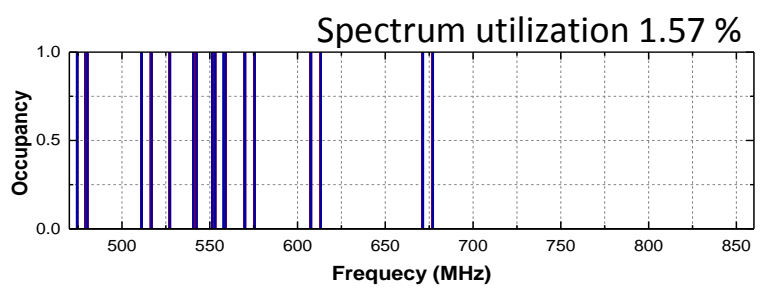

Fig 11: Calculated spectrum occupancy of UHF TV band with threshold $-60 \mathrm{dBm}$

\section{CONCLUSION}

UHF TV band spectrum sensor has been designed and implemented using a commercial TV box. Microcontroller is programmed to control the TV tuner for scanning the frequency band. This sensor has succeeded to sweep the UHF TV band spectrum within short time interval. The Microcontroller chip IC is programmed, to select frequency division references of $(31.25,50,62.5) \mathrm{kHz}$.

The UHF TV band spectrum has been recorded within 2 minutes interval using the system designed.

The measurements have been taken at the ASU (Ain Shams University) Faculty of Engineering building which roughly $4.5 \mathrm{~km}$ away from the TV transmitter station. From analyzing these data, the occupancy percentage at these conditions are determined and the spectrum utilization is obtained. The obtained results demonstrate a significant amount of unoccupied spectrum in the TV broadcasting band especially in range from (700-860) MHz region as there is no Digital TV broadcasting in EGYPT.

For future work, we plan to extend the measurement to cover the whole spectrum of the UHF and VHF bands. These future studies would help in more understanding of dynamic spectrum utilization and deployment of cognitive radio networks.

\section{ACKNOWLEDGMENTS}

The article is partially supported by a grant of the Foundation of Computer Science, NY, USA vide FCS/RT56/15

\section{REFERENCES}

[1] Federal Communication Commission, Third Report and Order and Memorandum Opinion and Order In the Matter of Unlicensed Operation in the TV Broadcast Bands, Additional Spectrum for Unlicensed Devices Below $900 \mathrm{MHz}$ and in the $3 \mathrm{GHz}$ Band, (2012).

[2] Shellhammer, S., Sadek, A., \& Zhang, W. (2009). Technical challenges for cognitive radio in the TV white space spectrum. Information Theory and Applications Workshop, 323-333.

[3] Baradkar, H., \& Akojwar, S. (2014). Implementation of Energy Detection Method for Spectrum Sensing in Cognitive Radio Based Embedded Wireless Sensor Network Node. Electronic Systems, Signal Processing and Computing Technologies (ICESC), 490-495.

[4] Abdulsattar, M., \& Hussein, Z. (2012). Energy Detection Technique for Spectrum Sensing in Cognitive Radio: A Survey. International Journal of Computer Networks \& Communications, 223-242.

[5] Yucek, T., \& Arslan, H. (2009). A Survey of Spectrum Sensing Algorithms For Cognitive Radio Applications. IEEE COMMUNICATIONS SURVEYS \& TUTORIALS, 116-130.

[6] Christopherclement, J., Krishnan, K., \& Bagubali, A (2012). Cognitive Radio: Spectrum Sensing Problems in Signal Processing. International Journal of Computer Applications, 37-40

[7] Tian, Z., \& Giannakis, G. (n.d.). A Wavelet Approach to Wideband Spectrum Sensing for Cognitive Radios. 2006 1st International Conference on Cognitive Radio Oriented Wireless Networks and Communications.

[8] Zhi, Q., Shuguang, C., Sayed, A., \& Poor, H. (2008). Wideband Spectrum Sensing in Cognitive Radio Networks. Communications, 2008. ICC '08. IEEE International Conference on.

[9] Nekovee, M. (n.d.). Cognitive Radio Access to TV White Spaces: Spectrum Opportunities, Commercial Applications and Remaining Technology Challenges. 2010 IEEE Symposium on New Frontiers in Dynamic Spectrum (DySPAN).

[10] Dzulkifli, M., Kamarudin, M., \& Rahman, T. (n.d.). Spectrum occupancy at UHF TV band for cognitive radio applications. 2011 IEEE International RF \& Microwave Conference.

[11] Khaira, N., \& Bhadauria, P. (2012). Cooperative spectrum sensing and Detection efficiency in cognitive radio network. International Journal of Electronics and Computer Science Engineering.

[12] Fawzy, A., Ghoname, R., \& Zekry, A. (2015). Developing front end wideband spectrum sensor for cognitive radio. International Journal of Sciences: Basic and Applied Research. 